\title{
Cerebral Salt Wasting in Traumatic Brain Injury Is Associated with Increased Morbidity and Mortality
}

This article was published in the following Dove Press journal:

Neuropsychiatric Disease and Treatment

\author{
Akella Chendrasekhar ${ }^{1,2}$ \\ Priscilla T Chow ${ }^{1,2}$ \\ Douglas Cohen' \\ Krishna Akella ${ }^{1,2}$ \\ Vinay Vadali, ${ }^{1,2}$ \\ Alok Bapatla ${ }^{1,2}$ \\ Jakey Patwari ${ }^{1}$ \\ Vladimir Rubinshteyn' \\ Loren Harris ${ }^{1,2}$ \\ 'Department of Surgery, Richmond \\ University Medical Center, Staten Island, \\ NY, USA; ${ }^{2}$ Department of Surgery, SUNY \\ Downstate Medical Center, Brooklyn, \\ NY, USA
}

\begin{abstract}
Introduction: In the setting of cerebral injury, cerebral salt wasting (CSW) is a potential cause of hyponatremia, which contributes to adverse effects and mortality.

Objective: The primary objective of this study was to evaluate the clinical outcomes of severe traumatic brain injury (TBI) patients complicated by CSW.

Methods: A retrospective data analysis was performed on data collected from patients with TBI with an abbreviated injury scale (AIS) greater than 3. Data was divided into 2 groups of patients with CSW and those without. The primary endpoint was incidence of adverse effects of CSW in regard to injury severity score (ISS), hospital length of stay (HLOS), ventilator days, ICU length of stay (ICU LOS) and survival to discharge. Data was analyzed using a one-way analysis of variance (ANOVA).
\end{abstract}

Results: A total of 310 consecutive patients with severe head injury (anatomic injury score 3 or greater) were evaluated over a 3 -year period. A total of 125 of the 310 patients $(40 \%)$ were diagnosed with cerebral salt wasting as defined by hyponatremia with appropriate urinary output and salt replacement. Patients with CSW had poorer outcomes in regard to ISS (21.8 vs 14.2, $\mathrm{p}<0.0001)$, HLOS (14.1 vs 3.5, $\mathrm{p}<0.0001$ ), ventilator days (5.0 vs 0.45 , $\mathrm{p}<0.0001)$, ICU LOS (8.5 vs $1.6, \mathrm{p}<0.0001)$, and survival to discharge $(88 \%$ vs $99 \%$, $\mathrm{p}<0.0001)$.

Discussion: Common adverse effects of CSW were noted in this study. Patients with TBI have a predilection towards development of CSW and consequently have poorer outcomes including increased morbidity and mortality. Data is sparse on the duration of CSW and degree of hyponatremia over time. Larger, comparative studies need to be performed to investigate the hyponatremic patient population and the clinical outcomes of those who present with CSW.

Keywords: hyponatremia, hypovolemia, Natriuresis, traumatic brain injury, neurosurgery

\section{Introduction}

Traumatic brain injury (TBI) is the most common cause of death in trauma. ${ }^{1}$ It is seen frequently in the setting of mechanisms of falls and motor vehicle accidents (MVAs). It was responsible for 2.8 million annual emergency department visits in the US in 2013 . ${ }^{1}$ Detailed outcome data regarding complicating sequelae, which can cause secondary brain injury is sparse. Hyponatremia is associated with worse outcomes in patients with TBI, ${ }^{2,3}$ however, data on the causes of acute hyponatremia is sparse. Two major differential diagnoses are commonly seen in TBI as causes of acute hyponatremia. They are syndrome of inappropriate antidiuretic hormone production (SIADH, hypervolemic/euvolemic hyponatremia) and cerebral salt wasting (CSW, hypovolemic hyponatremia). ${ }^{4}$ Both are equally well treated by using hypertonic saline as a resuscitative fluid. ${ }^{5}$ However, the use of hypertonic saline has its own detrimental
Correspondence: Akella Chendrasekhar Email achendrasekhar@rumcsi.org 
issues. ${ }^{5}$ The use of normal saline is more common but is only effective for the treatment of CSW not for SIADH. In patients with SIADH, a bolus of normal saline will fail to correct the hyponatremia and may worsen the hyponatremia due to Natriuresis continuing while diuresis is inhibited. ${ }^{6}$ Our ICU team had a protocol to manage all TBI patients complicated by hyponatremia with a one-liter bolus of normal saline as a diagnostic test. If the patient was diagnosed with CSW then we proceeded to treat the patients with salt supplementation (hypertonic saline/normal saline or salt tablets depending on clinical presentation). If SIADH was diagnosed, we proceeded to treat the patients with hypertonic saline.

\section{Materials and Methods}

We retrospectively performed a data analysis on severe TBI patients (abbreviated injury score to the head of 3 or greater) seen at our urban ACS verified Level 1 Trauma Center using our trauma registry and detailed review of electronic medical records. We studied the specific population of TBI patients complicated by acute hyponatremia (serum sodium less than $135 \mathrm{meq} / \mathrm{l}$ ) that had appropriate urine output and showed improved sodium levels when receiving a one-liter bolus of normal saline (TBI with hyponatremia presumed CSW). In our review, we excluded patients with chronic hyponatremia (previous admissions where serum sodium was low or history of severe congestive heart failure or severe liver cirrhosis or chronic renal failure). We also excluded severe TBI patients who died with 48 hrs of arrival in our ER as nonsurvivable. Once we identified the patients with TBI complicated by CSW, we compared this group to TBI patients without complicating CSW. Data obtained included age, injury severity score (ISS), abbreviated injury score for the head region (AIS-H), hospital length of stay (HLOS), ICU length of stay (ICU LOS), ventilator days and survival to hospital discharge. Data was analyzed using a one-way analysis of variance (ANOVA) using a commercially available statistical analysis software package (JMP). As this was a retrospective data analysis using de-identified data, we sought and obtained exemption from our institutional review board (SUNY Downstate Medical Center) for the study of human subjects. Therefore, no patient consent was required regarding patient confidentiality data. This is in compliance with the declaration of Helsinki.

\section{Results}

We identified 310 consecutive patients with severe TBI (AIS-H of 3 or greater) who were treated at our center between January 2016 and December 2018 (3-year period). One hundred and twenty-five of the 310 patients $(40 \%)$ had their hospital course complicated by CSW (Figure 1).

Thirty-two patients of the 310 patients with severe TBI were diagnosed with SIADH (10\%). The average age of the group of TBI patients complicated by CSW (61.5 years) was significantly higher than TBI patients not complicated by CSW (53.6 years, $\mathrm{p}=0.0028$ ) (Figure 2).

The degree of overall injury as documented by ISS was significantly greater in patients complicated by CSW (21.8

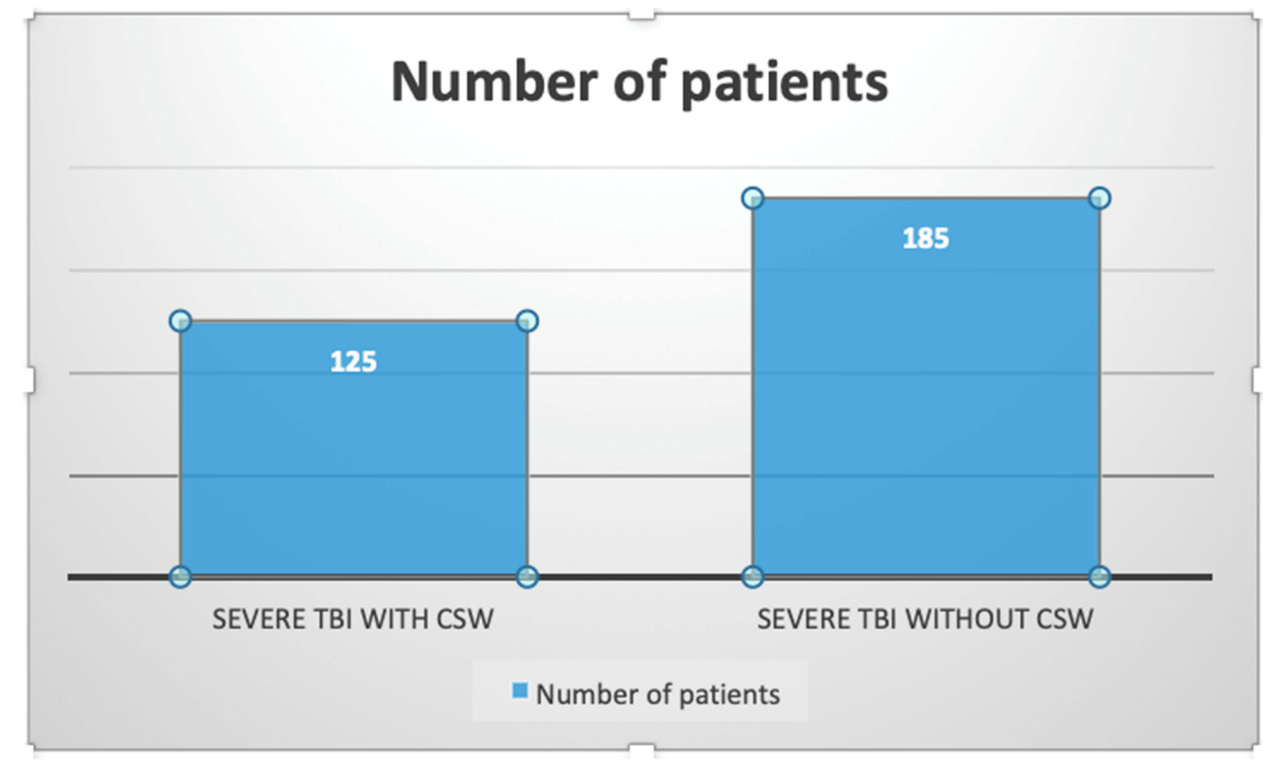

Figure I Number of Severe TBI Patients with and without CSW. 


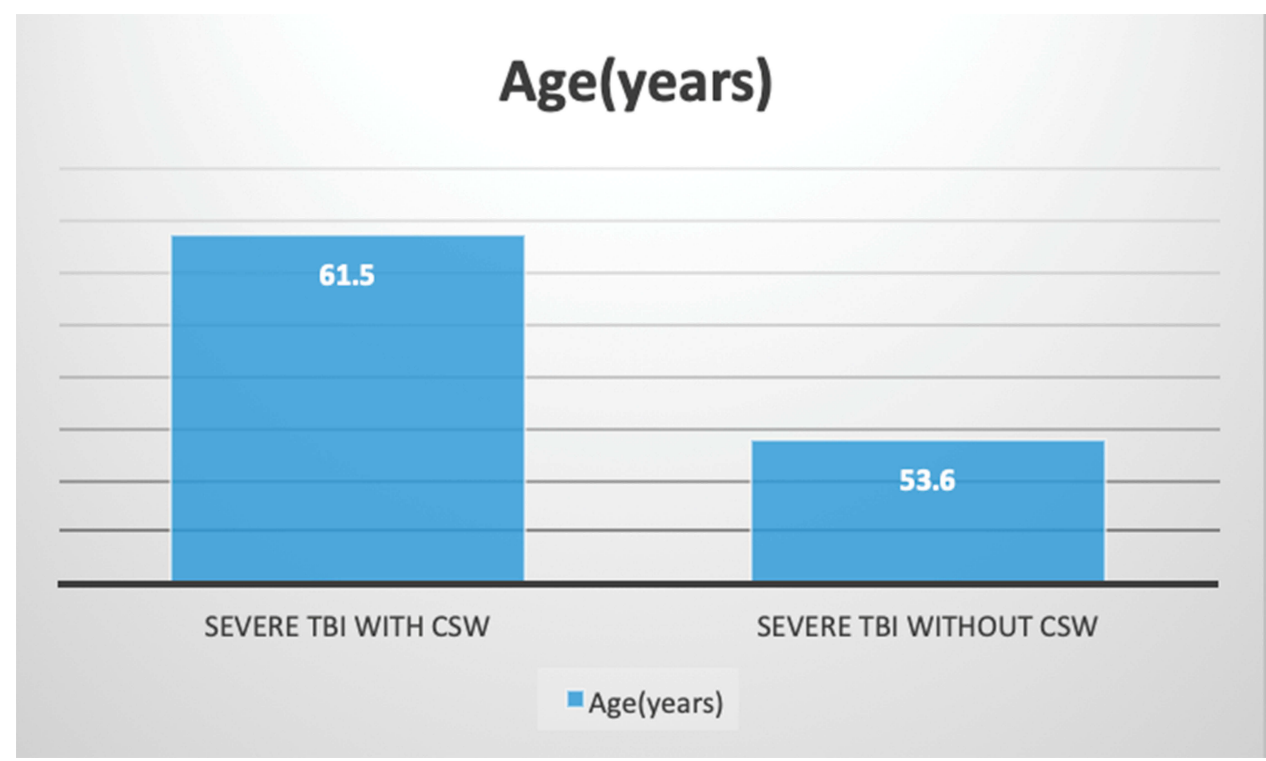

Figure 2 Age of Patients with Severe TBI with and without CSW.

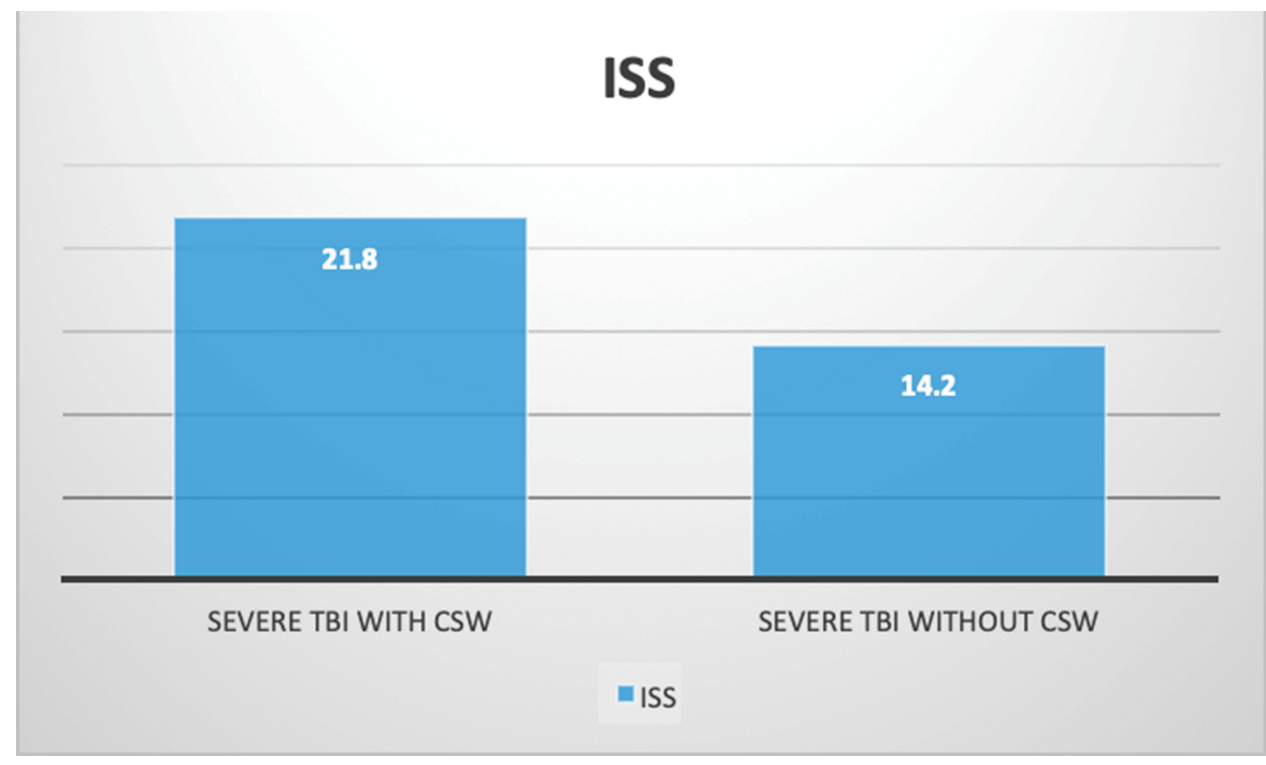

Figure 3 ISS for Severe TBI Patients with and without CSW.

vs $14.2, \mathrm{p}<0.0001)$. The AIS for the head region was not statistically different for the 2 groups (Figure 3 ).

Average Hospital length of stay (HLOS) was significantly longer in the CSW group as compared to patients without CSW (14.1 days vs 3.5 days, $p<0.0001$, Figure 4).

The ventilator days were also longer for the patients complicated by CSW (5.0 days vs 0.5 days, $\mathrm{p}<0.0001$ ). The ICU length of stay was also longer in the CSW complicated group ( 8.5 days vs 1.6 days, $\mathrm{p}<0.0001$, Figure 5).

Survival to discharge was also lower in the CSW group ( $88 \%$ vs $99 \%, p<0.0001$, Figure 6 ).
The patients who were complicated by SIADH had outcome data that was statistically no different than the TBI population without hyponatremia. The aforementioned data combined the SIADH complicated patients with the TBI patients without any hyponatremia as neither group had CSW.

\section{Discussion}

Severe TBI is a complex disease process with extensive variability depending on the location of the injury in the brain. ${ }^{7}$ Primary brain injury is avoided only through prevention methods such as wearing protective headgear as in 


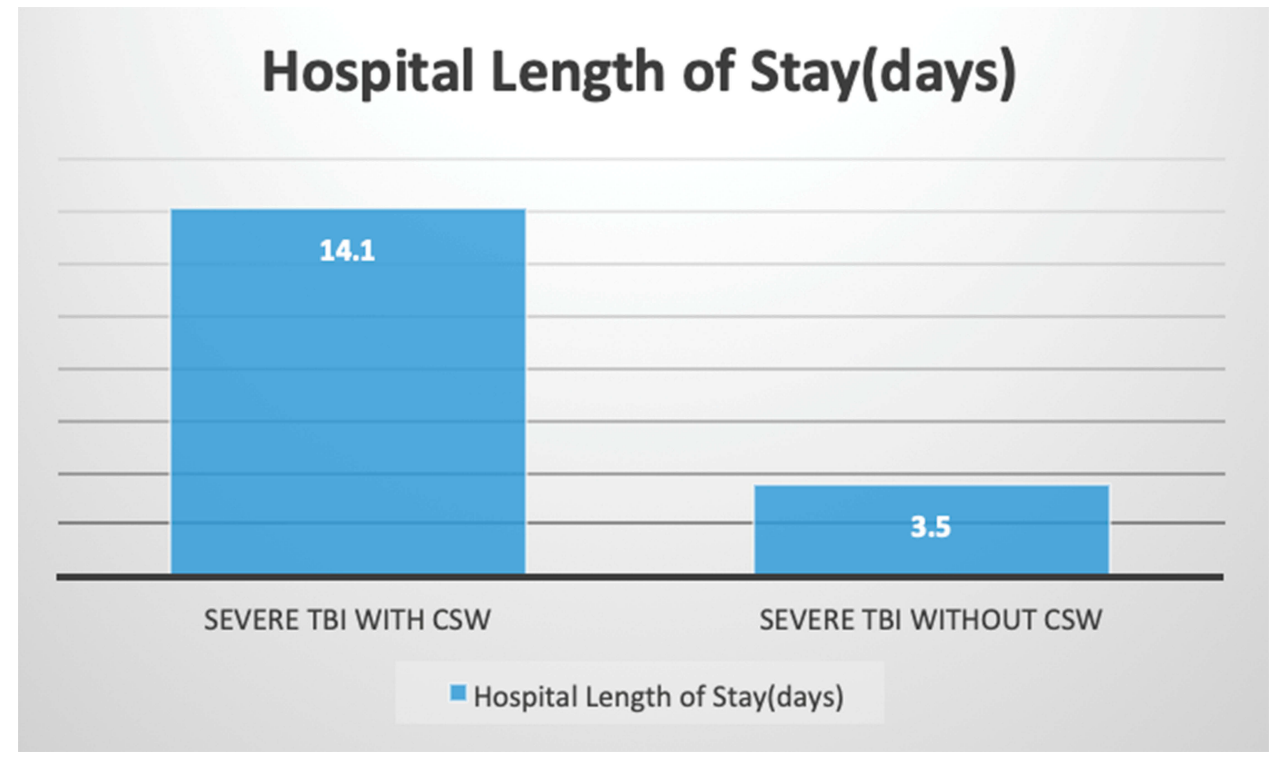

Figure 4 Hospital Length of Stay for Severe TBI Patients with and without CSW.

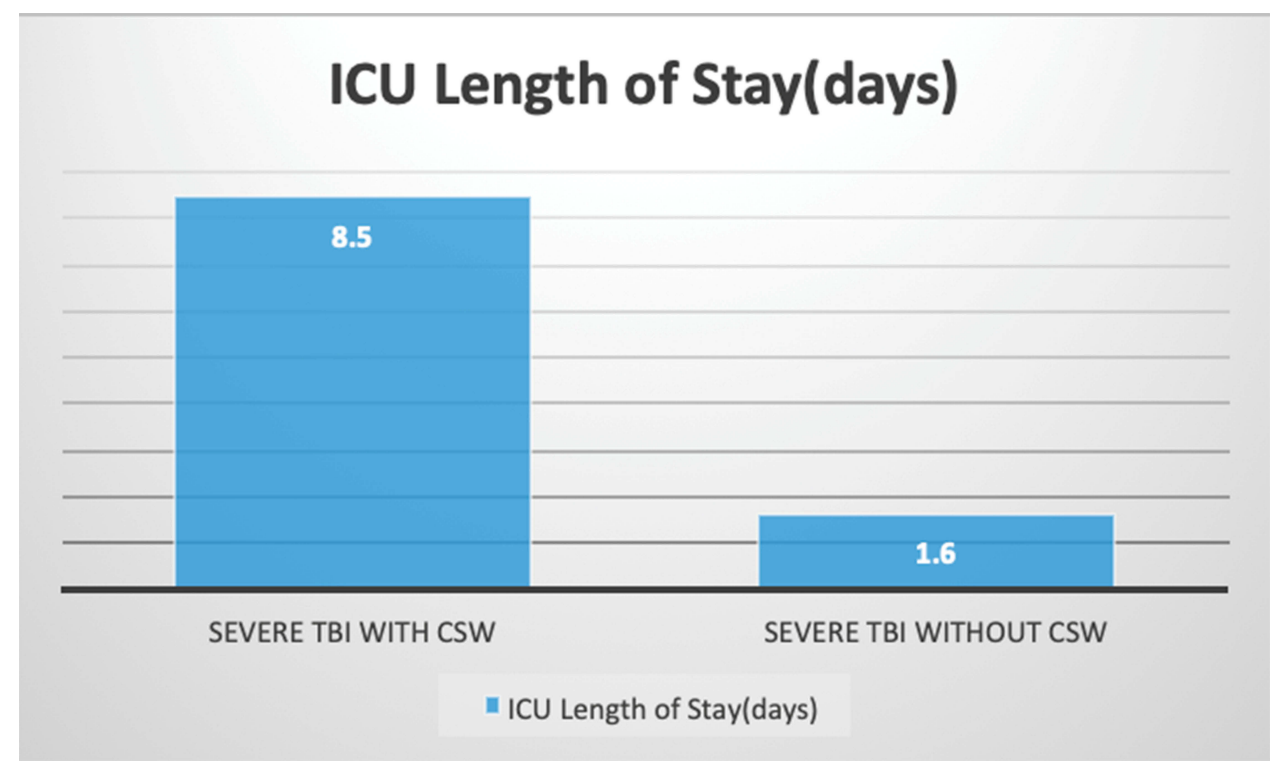

Figure 5 ICU Length of Stay for Severe TBI Patients with and without CSW.

the case of motorcycle helmets. ${ }^{8}$ Secondary brain injury is seen as a result of complicating issues such as hypotension, hypoxemia. ${ }^{9}$ The degree of brain injury becomes substantially greater and tracking and correcting the etiologies becomes much more important in the reduction or avoidance of secondary brain injury. ${ }^{9}$ Hyponatremia in severe traumatic head injury patients especially with traumatic subarachnoid hemorrhage is associated with increased brain swelling (secondary brain injury). ${ }^{5}$ We have previously published data on how hyponatremia (sodium level less than $135 \mathrm{meq} / \mathrm{l}$ ) is associated with increased mortality and morbidity. ${ }^{2}$ In that study, we did not breakdown the etiology of the hyponatremia. Hyponatremia however is a seemingly correctable cause of secondary injury. The rate of correction and degree of hyponatremia are also major issues. The correction can be as problematic as the hyponatremia (eg, central pontine myelinolysis) if it is done too rapidly. ${ }^{5}$ Most of our patients (113 out of 125) had hyponatremia in the range between $128 \mathrm{meq} / \mathrm{l}$ and $134 \mathrm{meq} / \mathrm{l}$ (90.4\%). Cerebral salt wasting has been described in the past as an unclear entity. ${ }^{6}$ But our data would suggest that given the current management 


\section{Survival to Hospital Discharge(\%)}

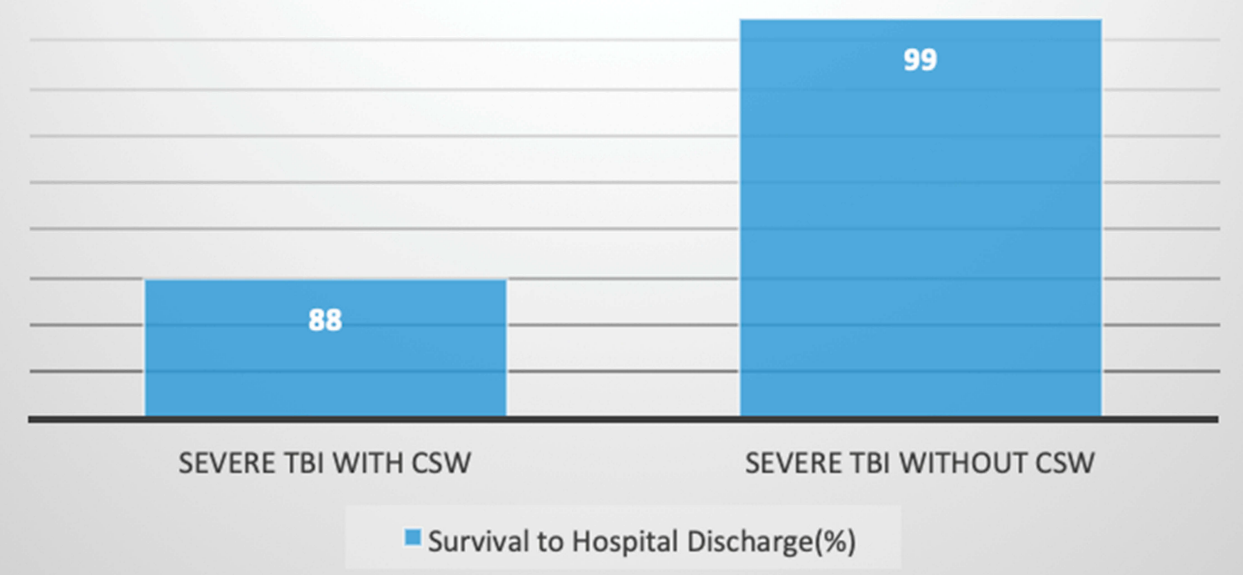

Figure 6 Survival to Hospital Discharge for Severe TBI Patients with and without CSW.

approaches to patients with severe TBI that CSW is much more common and associated with increased morbidity and mortality. The increased degree of injury associated with our CSW patients would suggest a possible reason why this group had a higher mortality, but of note, the AIS for the head region was not different which suggests that the degree of head injury was the same in the 2 groups. Increased age in this group could also contribute to diminished reserve resulting in increased complication rate which has been shown to correlate with increased length of stay. ${ }^{10}$ The correction of the hyponatremia due to CSW in our institution is dependent on patient symptoms. If the patient is asymptomatic the usual correction is to supplement the patient with PO salt tablets and track the correction. If the patient is symptomatic, then in conjunction with our neurosurgical colleagues, a decision is made whether we use normal saline solution or hypertonic saline $(3 \%)$ solution. Weaknesses in our study are retrospective nature of the data analysis and limitations of the type of head-injured patients seen at our trauma center. While AIS and ISS are important measures for degree of injury, they lack specificity of injury to be able to perform detailed comparisons. ${ }^{11}$ For example, severe injury to the cerebellar area has different consequences and outcomes than frontal injuries and AIS will not necessarily distinguish these differences.

CSW is clearly an important clinical entity that needs to be addressed and tracked by physicians caring for patients with severe TBI. Data is sparse on the duration of CSW and degree of hyponatremia over time and these are areas need to be addressed in the future research.

\section{Clinical Implications}

Older patients with TBI have a predilection towards development of CSW and consequently have poorer outcomes including greater HLOS, ICU LOS, ventilator days, and mortality.

\section{Ethics Statement}

SUNY Downstate Medical Center IRB determined that this study is exempt.

\section{Acknowledgment}

The abstract of this paper was presented at the 2019 CHEST Conference as a poster presentation discussion with interim findings. The poster's abstract was published in CHEST 2019 Annual Meeting Abstracts in CHEST Journal, Volume 156, Issue 4: https://journal.chestnet. org/article/S0012-3692(19)32544-9/fulltext.

\section{Disclosure}

The authors report no conflicts of interest in this work.

\section{References}

1. Taylor CA, Bell JM, Breiding MJ, et al. Traumatic brain injury related emergency department visits, hospitalizations, and deaths -United States, 2007 and 2013. MMWR Surveill Summ. 2017;66(9):1-16. doi:10.15585/mmwr.ss6609al

2. Smith M, Baltazar GA, Pate A, Akella K, Chendrasekhar A. Hyponatremia on initial presentation correlates with suboptimal outcomes after traumatic brain injury. Am Surg. 2017;83(4):e126-e128. 
3. Van Beek JGM, Mushkudiani NA, Steyerberg EW, et al. Prognostic value of admission laboratory parameters in traumatic brain injury: results from the IMPACT study. J Neurosurg. 2007;24:315-328.

4. Braun MM, Barstow CH, Pyzocha NJ. Diagnosis and management of sodium disorders: hyponatremia and Hypernatremia. Am Fam Physician. 2015;91(5):299-307.

5. Weismann D, Schneider A, Hoybye C. Clinical aspects of symptomatic hyponatremia. Endocr Connect. 2016;5(5):R35-R43. doi:10.15 30/EC-16-0046

6. Maesaka JK, Imbriano LJ, Miyawaki N. Determining fractional urate excretion rates in hyponatremic conditions and improved methods to distinguish cerebral/ renal salt wasting from the syndrome of inappropriate secretion of anti-diuretic hormone. Front Med (Lausanne). 2018;5:Article 319. doi:10.3389/fmed.2018.00319

7. Aerts H, Fias W, Caeyenberghs K, et al. Brain networks under attack: robustness properties and the impact of lesions. Brain. 2016;139(Pt 12):3063-3083. doi:10.1093/brain/aww194
8. Bonfield CM, Shin SS, Kanter AS. Helmets, head injury and concussion in sport. Phys Sportsmed. 2015;43(3):236-246. doi:10.1080/ 00913847.2015.1039922

9. Jeremitsky E, Omert L, Dunham CM, et al. Harbingers of poor outcome the day after severe brain injury: hypothermia, hypoxia, and hypoperfusion. J Trauma. 2003;54(2):312-319. doi:10.1097/01. TA.0000037876.37236.D6

10. Awad A, Bader-El-Den M, McNicholas J. Patient length of stay and mortality prediction: a survey. Health Serv Manag Res. 2017;30 (2):105-120. doi:10.1177/0951484817696212

11. Roswell SE, Barbosa RR, Diggs BS, et al. Specific abbreviated injury scale values are responsible for the underestimation of mortality in penetrating trauma patients by the injury severity score. $J$ Trauma. 2011;71(2 Suppl 3):S384-S388. doi:10.1097/TA.0b013e3182287c8d

\section{Publish your work in this journal}

Neuropsychiatric Disease and Treatment is an international, peerreviewed journal of clinical therapeutics and pharmacology focusing on concise rapid reporting of clinical or pre-clinical studies on a range of neuropsychiatric and neurological disorders. This journal is indexed on PubMed Central, the 'PsycINFO' database and CAS, and is the official journal of The International Neuropsychiatric Association (INA). The manuscript management system is completely online and includes a very quick and fair peer-review system, which is all easy to use. Visit http://www.dovepress.com/testimonials.php to read real quotes from published authors. 